\title{
PC Program for Analyzing One-Sample Longitudinal Data Sets Which Satisfy the Two-Stage Polynomial Growth Curve Model
}

\author{
THOMAS R. TEN HAVE, ${ }^{1}$ CHARLES J. KOWALSKI, ${ }^{2}$ AND \\ EMET D. SCHNEIDERMAN ${ }^{3}$ \\ Departments of ${ }^{1}$ Biostatistics and ${ }^{2}$ Oral Biology, University of Michigan, \\ Ann Arbor, Michigan 48109; ${ }^{3}$ Department of Oral and Maxillofacial \\ Surgery, Baylor College of Dentistry, Dallas, Texas 75246
}

\begin{abstract}
The two-stage polynomial growth curve model is described and a GAUSS program to perform the associated computations is documented and made available to interested readers. The two-stage model is similar to that considered by us earlier (Schneiderman and Kowalski: American Journal of Physical Anthropology 67:323-333, 1985; American Journal of Human Biology 1:31-42, 1989), i.e., it is appropriate for the analysis of one-sample longitudinal data collected at either equal or unequal time intervals. Here, however, the covariance matrix, $\Sigma$, instead of being considered arbitrary, is now assumed to have the special structure $\Sigma=W$ $\Lambda \mathbf{W}^{\prime}+\sigma^{2} \mathbf{I}$. We show the conditions under which this special structure may be expected to arise and how it may be exploited to produce sharper results in certain situations. The method and the program are illustrated and the results are contrasted to those obtained when $\Sigma$ is arbitrary. It is suggested that the two-stage model is more efficient when the same degree polynomial is adequate to model the data in the two situations, but that, should a higher degree be necessary for the two-stage model, confidence intervals and/or bands may be wider than those corresponding to $\Sigma$ arbitrary.
\end{abstract}

In an earlier paper (Schneiderman and Kowalski, 1985), we provided a SAS program for the analysis of one-sample longitudinal data sets assumed to satisfy Rao's (1959) polynomial growth curve model. More recently (Schneiderman and Kowalski, 1989), we implemented Hills' (1968) modification of Rao's procedure using GAUSS, a matrix programming language which runs on widely available $\mathrm{PC}$-compatible microcomputers. The first of these programs may be used to a) find the lowest degree polynomial in time adequate to fit the average growth curve $(\mathrm{AGC}), \mathrm{b})$ estimate and obtain confidence intervals for the coefficients of this polynomial, and c) construct confidence bands for the AGC. This program was limited to equally spaced time points. The second program accommodated unequally spaced time points and provided measures of growth velocity and acceleration for each of $\mathrm{N}$ individuals and for the sample as a whole.

The underlying model for the valid use of these procedures is that

$$
\begin{aligned}
& \mathbf{x}_{\mathrm{i}} \sim \operatorname{MVN}(\mathrm{W} \tau, \Sigma) \\
& \text { Tx1 TxP Px1 TxT }
\end{aligned}
$$

i.e., that the $\mathrm{T}$ observations, $\mathbf{x}_{\mathrm{i}}$, made at times $t_{1}, t_{2}, \ldots, t_{T}$ on individual $i(i=1$, $2, \ldots, \mathrm{N})$ follow a multivariate normal (MVN) distribution with mean or expected value $W_{\tau}$ and covariance matrix $\Sigma$. Here $W$ is the within-individual or time design matrix and $\tau^{\prime}=\left[\tau_{1}, \tau_{2}, \ldots, \tau_{p}\right]$ is the vector of regression coefficients for the $\mathrm{AGC} . \mathrm{W}$ is often taken to be

$$
\left[\begin{array}{cccc}
1 & t_{1} & \ldots & t_{1}^{D} \\
1 & t_{2} & \ldots & t_{2}^{D} \\
\cdot & \cdot & & \cdot \\
\cdot & \cdots & \ldots & \cdot \\
\cdot & \cdot & & \cdot \\
1 & t_{T} & \ldots & t_{T}^{D}
\end{array}\right]
$$

where $\mathrm{D}=\mathrm{P}-1$ is the degree of the polynomial being fit. Alternatively, $\mathrm{W}$ may contain the values of orthogonal or orthonormal polynomials (Ten Have et al., 1991). In this case we use the notation

Received June 11, 1990; accepted February 13, 1991.

Address reprint requests to Dr. Emet D. Schneiderman, Baylor College of Dentistry, 3302 Gaston Ave., Dallas, TX 75246. 


$$
\Phi=\left[\begin{array}{cccc}
1 & \phi_{1}\left(t_{1}\right) & \ldots & \phi_{\mathrm{D}}\left(t_{1}\right) \\
1 & \phi_{1}\left(t_{1}\right) & \ldots & \phi_{\mathrm{D}}\left(t_{2}\right) \\
\cdot & \cdot & & \cdot \\
\cdot & \cdot & \ldots & \cdot \\
1 & \phi_{1}\left(t_{\mathrm{T}}\right) & \ldots & \phi_{\mathrm{D}}\left(\mathrm{t}_{\mathrm{T}}\right)
\end{array}\right]
$$

and the expected value of $\mathbf{x}_{\mathrm{i}}$ is written $\mathrm{E}\left(\mathbf{x}_{\mathrm{i}}\right)=$ $\Phi \alpha$, the elements of $\alpha$ being referred to as orthogonal polynomial regression coefficients (OPRC's). When $\Phi$ is orthogonal, $\Phi^{\prime} \Phi$ is a diagonal matrix; when $\Phi$ is orthonormal, $\Phi^{\prime} \Phi==\mathrm{I}$, the identity matrix (see, e.g., Graybill, 1969).

While we have documented (Ten Have et al., 1991) that the use of $\Phi$ (and $\alpha$ ) has in general a number of advantages over $W$ (and $\tau)$, these are less compelling in the onesample case and we allow the user the use of either in our program. In what follows, we use $\mathrm{W}$ to refer to either 2 or 3 ; should it be necessary to distinguish between them, $\mathbf{W}$ and $\Phi$ will be used.

In Equation 1, $\Sigma$ is an arbitrary covariance matrix, i.e., it is estimated from the data without making any assumptions about its form. There are, however, situations in which one may reasonably assume some structure for $\Sigma$ and, when this is possible, more precise estimates of $\tau$ (and/or $\boldsymbol{\alpha}$ ) and narrower confidence bands for the AGC may be expected to result. One such situation arises when what has become known as the two-stage polynomial growth curve model is employed. This model was introduced by Rao (1965) and subsequently developed by several others, e.g., Fearn (1975, 1977), Ware and Wu (1981) and McMahan (1981). For a good overview, see Ware (1983).

In this paper we describe the two-stage polynomial growth curve model and provide a GAUSS program for testing whether or not it adequately fits a given one-sample longitudinal data set, for estimating and obtaining confidence intervals for its parameters, and for constructing confidence bands for the AGC. When the fit is adequate, more precise estimators and narrower confidence bands than those resulting from arbitrary $\Sigma$ may be anticipated. The method and the program are illustrated by several examples.

\section{THE TWO-STAGE MODEL}

The first stage structures the individuals' observations in terms of individual growth curve models. Specifically, it is assumed that

$$
\mathbf{x}_{\mathrm{i}} \mid \tau_{\mathrm{i}} \sim \operatorname{MVN}\left(\mathbf{W} \tau_{\mathrm{i}}, \sigma^{2} \mathbf{I}\right)
$$

where " $x_{i} \mid \tau_{i}$ " represents the conditional distribution of $x_{i}$ given $\tau_{i}$. In Equation 4 it is assumed that the deviations of the T observations on an individual from the growth curve particular to that individual are independent (i.e., $\sigma^{2} I$ is a diagonal matrix), but the reader should note, as will be made explicit below, it is not assumed that an individual's deviations from the AGC are independent. Otherwise stated, given $\tau_{i}$, i.e., if we knew the $\mathrm{i}^{\text {th }}$ individual's true growth curve, deviations from that curve may reasonably be assumed to be independent (due to measurement error and/or random fluctuations). This does not obviate the need for the inclusion of a covariance structure to model an individual's deviations from the AGC. It is this latter set of residuals which can be expected to be correlated. These correlations reflect, e.g., the tendency for an individual who is "small" at one time (as judged by his/her deviation from the AGC) to be "small" at successive points in time. The point being made here is that the oft-alluded to correlations among residuals in longitudinal data sets refer to the deviations from the $A G C$, not those from (known) individual-specific growth curves.

The second stage of the two-stage model expresses the relationship the individual growth curves and the AGC. It is predicated on the idea that in many situations one's prior (before looking at the data in hand) opinions about the $\tau_{i}$ will be exchangeable (i.e., that these opinions are the same for each of the individuals comprising the sample) and the fact that a convenient way to model exchangeability is to treat the $\tau_{j}$ as a random sample from some probability distribution. The mean of this distribution may then be interpreted as the vector of regression coefficients of the AGC, viz., $\tau$. The covariance matrix of this distribution, $\Lambda$, models the variability of the $\tau_{i}$ about $\tau$ and the covariability among the $\tau_{\mathrm{j}}$. Specifically, the second stage of the two-stage model is

$$
\tau_{\mathrm{i}} \sim \operatorname{MVN}(\tau, \Lambda)
$$

and this, together with Equation 4, implies that the (unconditional) distribution of the $x_{i}$ is

$$
\mathbf{x}_{\mathbf{i}} \sim \operatorname{MVN}\left(\mathbf{W}_{\tau}, \mathbf{W} \Lambda \mathbf{W}^{\prime}+\sigma^{2} \mathbf{I}\right) .
$$


Note that the $\mathbf{x}_{\mathrm{i}}$ are correlated (unless $\mathrm{W} \Lambda$ $W^{\prime}$ is diagonal) and that the model 6 differs from 1 only in that while $\Sigma$ is arbitrary in 1, it has a definite structure in 6 . It is this structure, ignored by the model 1 , which the two-stage model exploits (Fearn, 1975). Note also that the total variability in the $x_{j}$ has been conveniently partitioned by model 6 into between-individual variation $\left(\mathrm{W} \Lambda \mathbf{W}^{\prime}\right)$ and error variance $\left(\sigma^{2} I\right)$.

\section{TESTING FOR THE ADEQUACY OF THE TWO-STAGE MODEL}

As indicated above, if the two-stage model provides an adequate fit to the data, we can expect to obtain more precise estimates for its parameters than when $\Sigma$ is arbitrary. Following Rao (1965), it is convenient to test for the adequacy of the two-stage model in two steps. The first step tests that the mean of the $\mathbf{x}_{\mathrm{i}}$ is of the form $\mathrm{W} \tau$ without making any assumptions about the structure of $\Sigma$; the second step tests that $\Sigma=W \Lambda \mathbf{W}^{\prime}+\sigma^{2} \mathbf{I}$. The first test has already been described (Rao, 1959) and implemented (Schneiderman and Kowalski, 1985, 1989) and is based on the statistic

$$
\frac{(\mathrm{N}-\mathrm{T}+\mathrm{P}) \mathrm{N}}{(\mathrm{T}-\mathrm{P})(\mathrm{N}-1)}\left[\overline{\mathbf{x}}^{\prime} \mathbf{S}^{-1} \overline{\mathbf{x}}-\overline{\mathbf{x}}^{\prime} \mathbf{S}^{-1} \mathbf{W}^{\prime} \tilde{\tau}\right]
$$

which is distributed as $\mathrm{F}(\mathrm{T}-\mathrm{P}, \mathrm{N}-\mathrm{T}+\mathrm{P})$ under the null hypothesis $E\left(\mathbf{x}_{1}\right)=W \tau$. In statistic 7,

$$
\hat{\tau}=\left(W^{\prime} \mathbf{S}^{-1} \mathbf{W}\right)^{-1} W^{\prime} \mathbf{S}^{-1} \overline{\mathbf{x}}
$$

is the least-squares (and maximum likelihood) estimator of $\tau$ and $\bar{x}$ is the $T \times 1$ vector of means at each occasion.

The second test is based on

$$
\begin{aligned}
\lambda= & \frac{\left\{\left|\mathbf{W}^{\prime} \mathbf{W}\right||\mathbf{C}|\right\}^{(\mathbf{N}-1) / 2}}{\left|\mathbf{W}^{\prime} \mathbf{C W}\right|^{(\mathbf{N}-1) / 2}\left\{\frac{1}{\mathrm{~T}-\mathrm{P}} \operatorname{tr}[\mathbf{C}(\mathbf{I}\right.} \\
& \left.\left.\left.-\mathbf{W}\left(\mathbf{W}^{\prime} \mathbf{W}\right)^{-1} \mathbf{W}^{\prime}\right)\right]\right\}^{(\mathbf{N}-1)(\mathrm{T}-\mathbf{P}) / 2}
\end{aligned}
$$

and the fact that

$$
-2 \ln \lambda \sim \mathrm{X}^{2}\left[\frac{1}{2}(\mathrm{~T}-\mathrm{P})(\mathrm{T}+\mathrm{P}+1)-2\right]
$$

for large samples when $\Sigma=W \Lambda \mathbf{W}^{\prime}+\sigma^{2} \mathbf{I}$. In Equation 9, "I" is the determinant, "tr" the trace, and $\mathbf{C}=(\mathrm{N}-1) \mathrm{S}$ where $\mathrm{S}$ is the $\mathrm{T} \times \mathrm{T}$ sample covariance matrix of the $x$ 's. $C$ is sometimes called the (corrected) sums of squares and cross-products (SSCP) matrix. In (10), $X^{2}$ denotes the chi-squared distribution with the indicated number of degrees of freedom.

To perform either of the tests based on 7 or 9 , we need to specify the value of $D(=P-1)$, the degree of the polynomial being fit. We, following Rao (1959), approach this in a stepwise fashion. In 7 we start with $\mathrm{D}_{\mathrm{R}}$, a userspecified value of $D$ for the "reduced model" (often $D_{R}=1$ ) and repeat the test for all integer values of $D$ between $D_{R}$ and $D_{F}$, a user-specified value of $\mathrm{D}$ for the "full model" $\left(D_{R} \leqslant D_{F} \leqslant T-1\right)$, until this portion of the model is not rejected using the user-supplied level of significance (e.g., 0.05). We then use this value of $D$ in 9 , stepping up, if necessary, to $D_{\mathrm{F}}$ until this part of the model achieves an acceptable fit. If such a D exists, we call it the degree of the final polynomial model, viz., the smallest $D$ for which both parts of the two-stage model are adequately fit by the data. If there is no value of $D$ for which both tests prove nonsignificant, the two-stage model does not provide an adequate fit to the data and the user may wish to revert to the procedure appropriate for arbitrary $\Sigma$ (Schneiderman and Kowalski, 1985, 1989). If the user wishes to choose $D$ on grounds other than the step-up goodness-of-fit tests just described, he/she can simply specify $\mathrm{D}=$ $\mathrm{D}_{\mathrm{R}}=\mathrm{D}_{\mathrm{F}}$.

We should note that the test of $\Sigma=W \Lambda W^{\prime}$ $+\sigma^{2} I$ is related to fitting polynomials to the individual growth curves. As explained by Ware and Wu (1981), by finding the polynomial of lowest degree for which the test based on 9 fails to reject $\Sigma=\mathbf{W} \Lambda \mathbf{W}^{\prime}+\sigma^{2} \mathbf{I}$ as an adequate model, one chooses a polynomial family for the individual growth curves (also see Fearn, 1977). Thus the tests described above can be thought of in terms of first finding the smallest degree adequate to fit the AGC, and then determining the smallest degree required to fit each of the individual curves. This terminology is used in the interactive program described later. Tests for the adequacy of fit of the individual curves are equivalent to tests of $\Sigma=W \Lambda W^{\prime}+\sigma^{2} I$ for a given value of $D$.

Presuming that the two-stage model 
(Equation 6) is acceptable for some D, we proceed to estimate its parameters.

\section{ESTIMATION}

The least-squares estimators of the parameters in the two-stage model were given by Rao (1965) as--

$$
\begin{gathered}
\hat{\tau}_{\mathrm{i}}=\left(\mathbf{W}^{\prime} \mathbf{W}\right)^{-1} \mathbf{W}^{\prime} \mathbf{x}_{\mathrm{i}} \\
\hat{\tau}=\left(\mathbf{W}^{\prime} \mathbf{W}\right)^{-1} \mathbf{W}^{\prime} \overline{\mathbf{x}}=\frac{1}{\mathrm{~N}} \sum_{\mathrm{i}=1}^{\mathrm{N}} \hat{\tau}_{\mathrm{i}} \\
\hat{\boldsymbol{\sigma}}^{2}=\frac{1}{\mathrm{~N}(\mathbf{T}-\mathrm{P})} \\
\left.\times\left[\mathbf{C}+\mathbf{N} \overline{\mathbf{x x}}^{\prime}\right]\right\} \\
\hat{\Lambda}=\frac{1}{\mathrm{~N}-1}\left[\left(\mathbf{I}-\mathbf{W}\left(\mathbf{W}^{\prime} \mathbf{W}\right)^{-1} \mathbf{W}^{\prime}\right]\right. \\
\left.-(\mathbf{N}-1) \dot{\boldsymbol{\sigma}}^{2}\left(\mathbf{W}^{\prime} \mathbf{W}\right)^{-1}\right] .
\end{gathered}
$$

Notice that $\hat{\tau}$ as given in 12 assumes a simpler form than the $\hat{\tau}$ in 8 and is, in fact, the same as would be used in ordinary leastsquares regression problems (Draper and Smith, 1966 , p. 59). The $\hat{\tau}$ in 8 is often referred to as the "weighted estimator" (weighted by $\mathbf{S}^{-1}$ ); and the $\hat{\tau}$ in 12 is called the "unweighted estimator." Using this terminology, we can now document the sense in which the special structure $\Sigma=W \Lambda W^{\prime}+\sigma^{2} I$ is "exploited" by paraphrasing Chinchilli and Carter (1984) who noted that

$i$. the unweighted estimator is more efficient;

ii. fewer parameters need to be estimated for the covariance matrix in the two-stage model, so that there are more degrees of freedom for error and hence more powerful tests; and

iii. the unweighted estimator, unlike the weighted estimator, is normally distributed so that hypothesis testing, construction of confidence intervals, power calculations, etc., can be performed by standard multivariate normal techniques.

To elaborate on point ii, $\Lambda$ is $\mathrm{P} \times \mathrm{P}$, so the number of parameters in the covariance matrix for the two-stage model is $\mathrm{P}(\mathrm{P}+1) / 2+1$ (the distinct elements of $\Lambda$ and $\sigma^{2}$ ). If, on the other hand, the $\mathrm{T} \times \mathrm{T}$ matrix $\Sigma$ is arbitrary there are $\mathrm{T}(\mathrm{T}+1) / 2$ parameters to be estimated. Thus, e.g., if a line adequately fits $T$ $=5$ time points, there is a difference of $15-$
$4=11$ parameters to be estimated and hence a corresponding increase of 11 degrees of freedom when the two-stage model is employed.

In any event, our program provides estimates of the $\tau_{i}, \tau, \sigma^{2}$, and $\Lambda$. Having estimated these parameters, we next consider the construction of confidence intervals for the elements of $\tau$ and confidence bands for the AGC.

\section{CONFIDENCE INTERVALS}

Let $L$ be a $1 \times$ P vector of constants so that $L^{\prime} \tau$ is a linear function of $\tau$. Then Rao (1965) shows that a $(1-\alpha) \times 100 \%$ confidence interval for $L^{\prime} \tau$ is

$$
\mathbf{L}^{\prime} \dot{\tau} \pm\left[\frac{1}{\mathrm{~N}(\mathrm{~N}-1)} \mathbf{L}^{\prime} \mathbf{U} \mathbf{L}\right]^{1 / 2} \mathbf{t}_{\alpha / 2}
$$

where $\mathrm{U}=\left(\mathrm{W}^{\prime} \mathrm{W}\right)^{-1} \mathrm{~W}^{\prime} \mathrm{CW}\left(\mathrm{W}^{\prime} \mathrm{W}\right)^{-1}$ and $t_{\alpha / 2}$ is the upper $\alpha / 2$ point of the $t$-distribution with $\mathrm{N}-1$ degrees of freedom.

To obtain confidence intervals for the elements of $\tau$ we take, in succession,

$$
\begin{gathered}
\mathbf{L}_{1}^{\prime}=[1,0,0, \ldots, 0,0] \\
\mathbf{L}_{2}^{\prime}=[0,1,0, \ldots, 0,0] \\
\cdot \\
\dot{L}_{\mathbf{P}}^{\prime}=[0,0,0, \ldots, 0,1] .
\end{gathered}
$$

Noting that $\mathbf{L}_{\mathrm{i}}{ }^{\prime} \mathrm{UL}_{\mathrm{j}}=\mathrm{u}_{\mathrm{ii}}$, the $\mathrm{i}^{\text {th }}$ diagonal element of $\mathrm{U}, 15$ is most easily computed in the form

$$
\hat{\tau}_{\mathrm{i}} \pm \sqrt{\frac{\mathrm{u}_{\mathrm{ii}}}{\mathrm{N}(\mathrm{N}-1)}} \mathbf{t}_{\alpha / 2} .
$$

To obtain confidence bands for the AGC we use

$$
\mathbf{L}_{\mathbf{i} \tau}^{\prime} \pm \sqrt{\frac{\overline{\mathrm{P}}}{\mathrm{N}(\mathrm{N}-\mathrm{P})} \mathbf{L}_{\mathrm{i}}^{\prime} \mathbf{U L}_{\mathrm{i}} \mathbf{F} \alpha}
$$

where $\mathrm{F}$ has $\mathrm{P}$ and $\mathrm{N}-\mathrm{P}$ degrees of freedom and the $\mathrm{L}_{\mathrm{i}}{ }^{\prime}$ are identified with the T rows of W, viz.,

$$
\begin{aligned}
& \mathrm{L}_{1}{ }^{\prime}=\left[1, \mathrm{t}_{1}, \ldots, \mathrm{t}_{1}{ }^{\mathrm{D}}\right] \\
& \mathrm{L}_{2}^{\prime}=\left[1, \mathrm{t}_{2}, \ldots, \mathrm{t}_{2}{ }^{\mathrm{D}}\right] \\
& \cdot \\
& \dot{\mathrm{L}_{\mathrm{T}}}
\end{aligned}
$$


When these are used successively in 17 , we obtain simultaneous $(1-\alpha) \times 100 \%$ confidence intervals for the AGC at each of the T time points and these may be joined together (Rao, 1959) to obtain a confidence band for the AGC.

We turn now to a description of our program. Details concerning the equipment required to run the program and how to obtain a copy of it are given in Appendix A. A step-by-step illustration of its use is given in Appendix B. It will be noted that the program is completely interactive. The user is prompted for all the information needed to perform the analysis.

\section{THE PROGRAM}

The GAUSS program, called 2STG, determines the degrees of the polynomials necessary to fit the AGC and the individual growth curves, estimates and provides confidence intervals for the regression coefficients of the final model, determines a confidence band for the AGC, and plots the $A G C$ and its confidence band. The user may select from four different forms of the time design matrix, $W$, viz., integer orthogonal scores, non-integral orthogonal scores, orthonormal scores or the original time scores (Eq. 2). The first of these may be selected only when the times of measurement are equally spaced and separated by one unit. The program can read the $\mathrm{N} \times \mathrm{T}$ data matrix, $\mathrm{X}$, from either an ASCII file or a GAUSS data set. One need not have installed GAUSS to run the program.

The program is invoked by the command

\section{GAUSSRUN 2STG}

The user is then asked if the data are in an ASCII file or a GAUSS data set. If the data are in an ASCII file, then the program prompts the user for the name of the file, the number of subjects (rows), and the number of time points (variables or columns). If the data are in a GAUSS data set, the program asks for the name of the GAUSS file and the variable numbers corresponding to the first and last response variables.

The program then prompts the user for the following information regardless of the type of data set input:

1. the degree of the reduced polynomial model $\left(\mathrm{D}_{\mathrm{R}}\right)$
2. the degree of the full model $\left(D_{F}\right)$;

3. the numerical values of the time points (one per line) or the default, consecutive integers beginning with one;

4. a value indicating which of the four forms of the time design matrix $W$ should be used:

non-integer orthogonal scores (1), integer orthogonal scores for equally spaced time points (2),

orthonormal scores (3), original time points (4);

5. the level (e.g., 0.95) of the confidence band for the AGC; and

6. the $P$ value (e.g., 0.05 ) above which models are considered to fit the data adequately.

The program then prints the time design matrix $W$ and the values of the estimated regression coefficients corresponding to the full polynomial model for each individual in the sample. It then tests the adequacy of the reduced model and each higher degree polynomial model until one satisfies the userprovided $P$ value criterion. This is then the degree of the AGC. If no model with degree less than that of the full model fits the AGC, the program stops.

If a suitable model for the AGC is found, the program then tests whether or not this model or any higher degree model (less than that of the full model) fits the individual curves. The lowest degree model that satisfies the user-specified $P$ value criterion for the individual curves is then the final model used in subsequent analyses. If no model with degree less than the full model fits the individual curves, the program stops.

The coefficients of the individual and average curves based on the final model are displayed, as well as the covariance matrix of the coefficients of the AGC and the error variance, $\hat{\boldsymbol{\sigma}}^{2}$.

The program also checks if the covariance matrix of the coefficients, $\hat{\Lambda}$, is positive semidefinite. If it is not, the program computes a modified estimate due to Carter and Yang (1986) which is guaranteed to be positive semi-definite.

The final statistical output consists of two tables containing the standard errors and confidence intervals for the coefficients; and the confidence bands for the AGC at each time of measurement.

Finally, the program generates plots of the fitted AGC, its confidence band and the ob- 
served AGC. The program allows the user to change the title and axis labels of the plots.

An example session is detailed in Appendix B. Some remarks concerning the example are given below.

\section{THE EXAMPLE SESSION}

The example session is based on the wellknown data set containing the values of ramus heights of $\mathrm{N}=20$ boys at ages $8.0,8.5$, 9.0 and 9.5 years of age, which has been analyzed, among others, by Elston and Grizzle (1962), Rao (1965), Grizzle and Allen (1969), and Goldstein (1979, p. 92). For the convenience of those requesting a copy of 2STG, these sample data are supplied in an ASCII file along with the 2STG program. For purposes of the example in Appendix B, these data are in an ASCII data set (located in the same directory from which 2STG is invoked), $\mathrm{N}=20, \mathrm{~T}=4$; we specify $\mathrm{D}_{\mathrm{R}}=1$, $\mathrm{D}_{\mathrm{F}}=4$; the time points are coded $\mathrm{t}=1$, $2, \ldots, 5$; the $\mathrm{W}$ form of the time design matrix is employed; we choose to obtain $95 \%$ confidence intervals and bands; and to perform the goodness-of-fit tests at the 5\% level of significance. The entire session is reproduced in Appendix B: Prompts, responses and output are shown. User responses are enclosed in brackets; \{\} . The non-graphical output appears on the screen and is sent to a file (automatically created) called 2STG.OUT from which it may be subsequently printed. The graphical output also appears on the screen and may be plotted by pressing the space bar at any time during the graphics session. The three publicationquality graphs produced directly by the program on a laser printer are shown in Figure 1.

\section{RESULTS}

It is seen that $\mathrm{E}\left(\mathbf{x}_{\mathrm{i}}\right)=\mathrm{W}_{\tau}$ is fit adequately with $\mathrm{D}=1(P=0.9096)$, and that $\mathrm{D}=1$ also suffices for the individual curves $(P=$ 0.1078 ). Thus the degree of the final polynomial model is $\mathrm{D}=1$ and the $\tau_{i}, \tau, \boldsymbol{\sigma}^{2}$ and $\Lambda$ are all estimated on that basis. The Carter and Yang (1986) modification of $\Lambda$ is not necessary in this example.

A comparison of the lengths of the confidence intervals for the elements of $\tau$ and the confidence bands for the AGC at the four times of measurement obtained from 2STG and for arbitrary $\Sigma$ (Schneiderman and Kowalski, 1985) is given in Table 1. It is seen
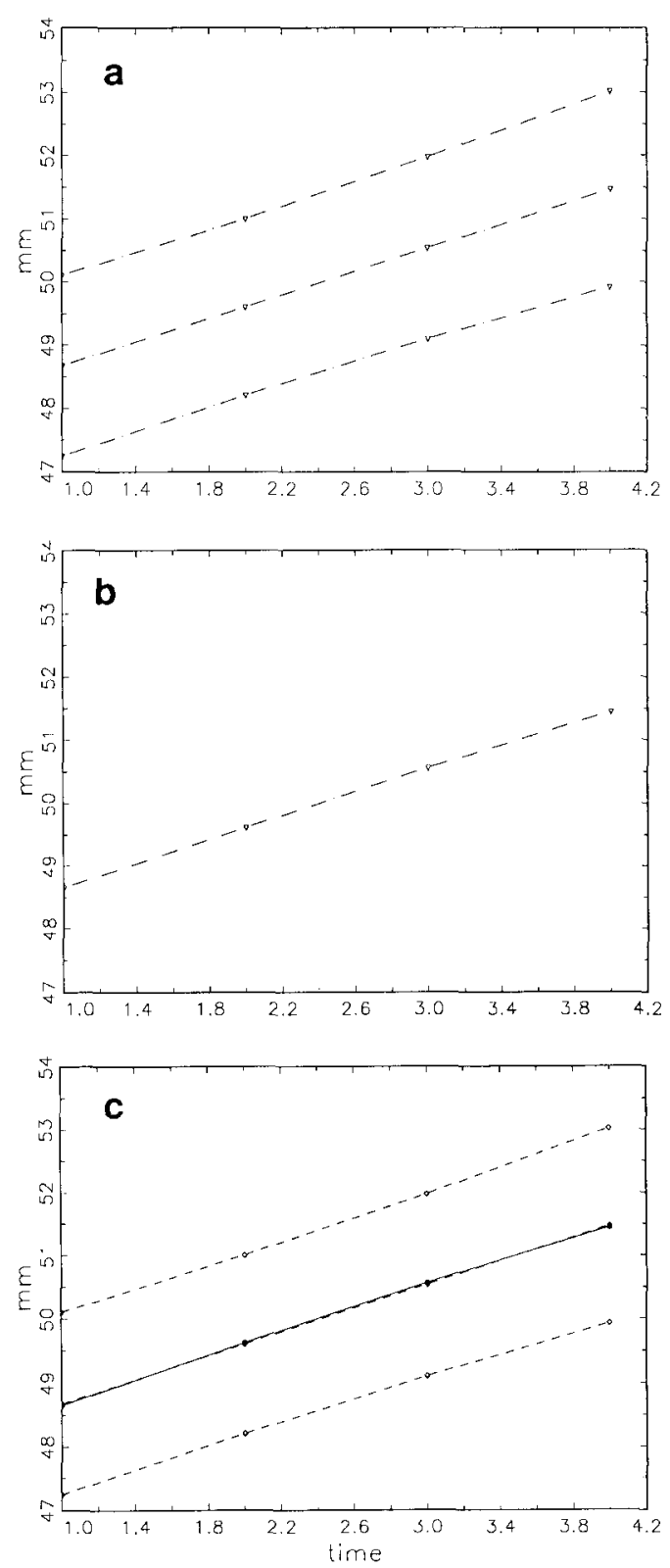

Fig. 1. The three plots showing human mandibular ramus growth (data from Elston and Grizzle, 1962) generated by the program. The four time-points are at half-year intervals, from 8 to 9.5 years of age. Plot a shows the fitted average growth curve and its associated $95 \%$ confidence band. Plot $b$ shows the average curve based on the original unfitted means and Plot c shows the observed curve superimposed on the fitted curve and its confidence band. 
TABLE 1. Confidence intervals for the parameters and confidence bands for the AGC for different assumptions concerning $\Sigma$

\begin{tabular}{lrr}
\hline & Arbitrary $\Sigma$ & $\Sigma=W \Lambda W^{\prime}+\sigma^{2} I$ \\
\hline$\tau_{1}$ & $47.723 \pm 1.425$ & $47.743 \pm 1.281$ \\
$\tau_{2}$ & $0.931 \pm 0.254$ & $0.933 \pm 0.273$ \\
Time 1 & $48.653 \pm 1.635$ & $48.676 \pm 1.433$ \\
Time 2 & $49.584 \pm 1.614$ & $49.609 \pm 1.397$ \\
Time 3 & $50.515 \pm 1.650$ & $50.542 \pm 1.437$ \\
Time 4 & $51.446 \pm 1.739$ & $51.475 \pm 1.547$ \\
\hline
\end{tabular}

that the half-width of the confidence interval from 2STG is shorter for $\tau_{1}$, but somewhat longer for $\tau_{2}$. The widths of the confidence bands from 2STG are shorter at each time of measurement. This illustrates that while the two-stage model "does better" in most respects than the model based on arbitrary $\Sigma$, this advantage is not uniform, not all the confidence intervals based on 2STG need be smaller than those produced when $\Sigma$ is considered arbitrary.

\section{DISCUSSION}

We have seen that while the two-stage model can be expected to produce sharper results when its assumptions are satisfied, this expectation is not necessarily realized for all the confidence intervals and/or confidence bands in particular instances. It should also be realized that if a higher degree polynomial than that adequate to model the $\mathrm{AGC}$ is required to fit the individual curves, the two-stage model may even do worse than the model based on arbitrary $\Sigma$. We show this using the data previously considered by Schneiderman and Kowalski (1985, 1989). There we had $\mathrm{N}=12, \mathrm{~T}=5$ and showed that the model $\mathrm{E}\left(\mathbf{x}_{\mathrm{i}}\right)=\mathrm{W} \tau$ was fit adequately for $\mathrm{D}=2(P=0.14)$. However, using 2STG, the hypothesis $\Sigma=W \Lambda W^{\prime}+\sigma^{2} \mathbf{I}$ is not accepted until $\mathrm{D}=3$. The degree of the final polynomial model is then $\mathrm{D}=3$ and all the parameters of the model are estimated on this basis.

A comparison of the lengths of the confidence intervals for the elements of $\tau$ corresponding to several situations is given in Table 2. The confidence intervals are presented for

i. $\Sigma$ arbitrary with $\mathrm{D}=2$;

ii. $\Sigma=W \Lambda W^{\prime}+\sigma^{2} I$ with $D=3$;

iii. $\Sigma=W \Lambda W^{\prime}+\sigma^{2} I$ with $D=2$.
TABLE 2. Confidence intervals for the regression coefficients for different assumptions concerning $\Sigma$ and values of $D$

\begin{tabular}{lcrr}
\hline & $\begin{array}{c}\text { Arbitrary } \Sigma \\
(\mathrm{D}=2)\end{array}$ & $\begin{array}{c}\text { 2STG } \\
(\mathrm{D}=3)\end{array}$ & \multicolumn{1}{c}{ 2STG } \\
& $(\mathrm{D}=2)$ \\
\hline$\tau_{1}$ & $18.557 \pm 2.477$ & $21.062 \pm 2.991$ & $18.868 \pm 1.583$ \\
$\tau_{2}$ & $8.819 \pm 1.856$ & $5.209 \pm 3.936$ & $8.290 \pm 1.342$ \\
$\tau_{3}$ & $-0.820 \pm 0.272$ & $0.453 \pm 1.303$ & $-0.722 \pm 0.198$ \\
$\tau_{4}$ & - & $-0.131 \pm 0.132$ & - \\
\hline
\end{tabular}

TABLE 3. Confidence bands for the AGC for different assumptions concerning $\Sigma$ and values of $D$

\begin{tabular}{lccc}
\hline Time & $\begin{array}{c}\text { Arbitrary } \Sigma \\
(\mathrm{D}=2)\end{array}$ & $\begin{array}{c}\text { 2STG } \\
(\mathrm{D}=3)\end{array}$ & $\begin{array}{c}\text { 2STG } \\
(\mathrm{D}=2)\end{array}$ \\
\hline 1 & $26.556 \pm 1.658$ & $26.593 \pm 1.498$ & $26.436 \pm 1.249$ \\
2 & $32.916 \pm 1.462$ & $32.246 \pm 2.367$ & $32.560 \pm 1.539$ \\
3 & $37.636 \pm 2.247$ & $37.238 \pm 2.762$ & $37.239 \pm 2.224$ \\
4 & $40.716 \pm 2.874$ & $40.788 \pm 3.154$ & $40.475 \pm 2.688$ \\
5 & $42.157 \pm 3.560$ & $42.109 \pm 3.972$ & $42.226 \pm 3.143$ \\
\hline
\end{tabular}

The third of these is included to allow a comparison between arbitrary $\Sigma$ and the two-stage model of the same degree (recall that this can be done in 2STG by taking $\mathrm{D}_{\mathrm{R}}=$ $D_{F}=2$ ). It is seen that the lengths of the $95 \%$ confidence intervals for the two-stage model with $\mathrm{D}=3$ are actually longer than those corresponding to arbitrary $\Sigma$. When the models are compared using the same $\mathrm{D}=2$, the two-stage model produces shorter confidence intervals.

Table 3 contains analogous information pertaining to confidence bands for the AGC at times $\mathrm{t}=1,2, \ldots, 5$. Again, these are generally shortest for the two-stage model with $\mathrm{D}=2$, of intermediate length for arbitrary $\Sigma$, and longest for the two-stage model with $\mathrm{D}=3$.

We might also note that in this example the estimator of $\Lambda$ was not positive semidefinite and so the modification due to Carter and Yang (1986) was employed. They suggested that when this modification is necessary, "... one should question the validity of the model specification.", i.e., one might use this as an informal test for the goodness-of-fit of the two-stage model. In the current example, this might lead one to revert to methods appropriate for arbitrary $\Sigma$ simply on this basis.

In any event, we have now described the two-stage polynomial growth curve model, documented some of its properties, provided 
a program to perform the computations, and illustrated the method, the program and some of the modeling problems users may expect to encounter in practice. We believe that the two-stage model is conceptually sound and has a number of potential advantages; its use should be considered whenever such use can be justified, especially in situations where the same degree polynomial may be used as when $\Sigma$ is considered arbitrary (i.e., if the same degree suffices for both the $A G C$ and the individual growth curves). If a higher degree polynomial is required to adequately fit the data, the two-stage model may not be more efficient in estimating the AGC.

\section{ACKNOWLEDGMENTS}

This research was supported by grant DE08730 from the National Institute of Dental Research.

\section{LITERATURE CITED}

Carter, RL, and Yang MCK (1986) Large-sample inference in random coefficient regression models. Communications Stat. Theory Methods 8:2507-2526.

Chinchilli VM, and Carter WH (1984) A likelihood ratio test for a patterned covariance matrix in a multivariate growth-curve model. Biometrics 40:151-156.

Draper N, and Smith H (1966) Applied Regression Analysis. New York: Wiley.

Elston RC, and Grizzle JE (1962) Estimation of timeresponse curves and their confidence bands. Biometrics $18: 148-159$

Fearn T (1975) A Bayesian approach to growth curves. Biometrika 62:89-100.

Fearn T (1977) A two-stage model for growth curves which leads to Rao's covariance adjusted estimators. Biometrika 64:141-143.

Goldstein H (1979) The Design and Analysis of Longitudinal Studies, New York: Academic Press.

Graybill FA (1969) Introduction to Matrices with Applications in Statistics. Belmont, CA: Wadsworth.

Grizzle JE, and Allen, DM (1969) Analysis of growth and response curves. Biometrics 25:307-318.

Hills M (1968) A note on the analysis of growth curves. Biometrics 24:189-196.

McMahan CA (1981) An index of tracking. Biometrics $37: 447-455$.
Rao CR (1959) Some problems involving linear hypotheses in multivariate analysis. Biometrika 46:49-58.

Rao CR (1965) The theory of least squares when the parameters are stochastic and its application to the analysis of growth curves. Biometrika 52:447-458.

Schneiderman ED, and Kowalski CJ (1985) Implementation of Rao's one-sample polynomial growth curve model using SAS. Am. J. Phys. Anthropol. 67:323-333.

Schneiderman ED, and Kowalski CJ (1989) Implementation of Hills' growth curve analysis for unequal-time intervals using GAUSS. Am. J. Hum. Biol. 1:31-42.

Ten Have TR, Kowalski CJ, and Schneiderman ED (1991) A GAUSS program for obtaining orthogonal polynomial regression coefficients for use in polynomial regression analysis. Am. J. Hum. Biol. submitted for publication.

Ware JH (1983) Growth curves. In S Kotz, NL Johnson, and CB Read (eds): Encyclopedia of Statistical Sciences. New York: Wiley, pp. 539-542.

Ware JH, and Wu MC (1981) Tracking: Prediction of future values from serial measurements. Biometrics $37: 427-437$

\section{APPENDIX A: COMPUTER IMPLEMENTATION}

This program can be obtained on a 5.25 in double-density floppy disk by sending $\$ 10$ to defray the cost of handling and licensing fees. The program will run on a IBM-PC/XT or AT compatible computer. The computer must be equipped with a numerical coprocessor from the 8087 family and $640 \mathrm{~K}$ of memory. The computer must be configured so that at least $430 \mathrm{~K}$ of memory is available, i.e., not tied up with memory resident programs such as Windows. EGA or VGA graphics capability is required to display the color graphics. No additional software is required (other than what one would normally use to enter a data set); run-time modules are supplied with the program so that no compiler or interpreter is necessary. The program, written in GAUSS, version 2.0, revision 20, requires no additional installation or modification, and is run with a single command. When requesting the program, address inquiries to EDS and make checks payable to Baylor College of Dentistry. 


\section{APPENDIX B. AN EXAMPLE RUN}

Estimation of two-stage polynomial models (2STG) by E. D. Schneiderman, C. J. Kowalski \& T. R. Ten Have

Output is going to 2STG.OUT

Date: $5 / 23 / 90$

Time: 12:20:47

Do you want to use a GAUSS or ASCII data set? Enter G or A: $\{A\}$

*** ASCII DATA SET INPUT ***

NOTE - DATA SET MUST BE A MATRIX WHERE EACH SUBJECT IS A ROW AND EACH COLUMN IS A TIME-POINT

YOU MUST KNOW THE NUMBER OF SUBJECTS \& TIME POINTS TO PROCEED!

ALSO BE SURE TO INCLUDE THE EXTENSION OF THE FILE NAME (E.G. RAMUS.ASC)

Enter ASCII data set containing observations: \{HRAMUS.ASC

Enter number of SUBJECTS (N) in hramus.asc: $\{20\}$

Enter number of TIME-POINTS (T) in hramus.asc: $\{4\}$

Enter degree of reduced model (RD): $\{1\}$

Enter degree of full model (FD): $\{3\}$

Do you want to enter the values of the time variable? (Enter ' $\mathrm{Y}$ ' or ' $N$ ')

(If no, the program will provide consecutive integers beginning with one for the time variable)

$\{\mathrm{N}\}$

For the time design matrix (W),

Enter: 1 if non-integer orthogonal scores

2 if integer orthogonal scores

(inputted scores must be integers one unit apart)

3 if orthonormal scores

$\{4\}$

4 if original time scores

Enter the level (e.g. .90) for the confidence band of the average growth curve and

confidence intervals for parameters:

$\{.95\}$

Enter p-value for test of lack of fit of polynomial models:

$\{.05\}$

Time design matrix

$\begin{array}{rrrr}1.00 & 1.00 & 1.00 & 1.00 \\ 1.00 & 2.00 & 4.00 & 8.00 \\ 1.00 & 3.00 & 9.00 & 27.00 \\ 1.00 & 4.00 & 16.00 & 64.00\end{array}$

Press $<$ ENTER $>$ to continue

FULL MODEL COEFFICIENTS

Press $<$ ENTER $>$ to continue

$\begin{array}{rrrr}44.70 & 4.583 & -1.700 & 0.2167 \\ 44.20 & 3.117 & -1.050 & 0.1333 \\ 47.10 & -1.717 & 1.050 & -0.1333 \\ 45.80 & -1.250 & 0.6000 & -0.05000\end{array}$




$\begin{array}{lccc}45.70 & 2.567 & -0.7500 & 0.08333 \\ 50.30 & 3.250 & -1.200 & 0.1500 \\ 49.50 & 1.450 & 0.3500 & -0.1000 \\ 47.70 & 3.717 & -1.950 & 0.3333 \\ 42.40 & 7.800 & -2.400 & 0.3000 \\ 38.90 & 8.950 & -3.250 & 0.4000 \\ 50.90 & 0.3833 & -0.1000 & 0.01667 \\ 55.30 & -12.02 & 5.950 & -0.7333 \\ 51.40 & 0.7667 & -0.1000 & 0.03333 \\ 46.80 & 1.883 & -0.5500 & 0.06667 \\ 49.00 & 0.4333 & 0.2000 & -0.03333 \\ 50.10 & 0.2667 & 0.4000 & -0.06667 \\ 46.70 & 0.5667 & -0.1000 & 0.03333 \\ 50.70 & 3.417 & -0.9000 & 0.08333 \\ 43.80 & 3.083 & -0.7500 & 0.06667 \\ 53.00 & -12.57 & 6.800 & -0.9333\end{array}$

Press $<$ ENTER $>$ to continue

**** TEST FOR ADEQUACY OF FIT OF DEGREE 1 OF THE AVERAGE CURVE **** $\mathrm{F}(2,18)=0.09527 \quad \mathrm{p}=0.9096$

Press $<$ ENTER $>$ to continue

DEGREE OF FINAL AVERAGE POLYNOMIAL MODEL: $\quad 1.000$

Press $<$ ENTER $>$ to continue

**** TEST FOR ADEQUACY OF FIT OF DEGREE 1 OF THE INDIVIDUAL CURVES ****

LAMBDA $=0.01093 \quad$ CHISQUARE WITH 9 DF $=9.032 \quad \mathrm{p}=9.1078$

Press $<$ ENTER $>$ to continue

DEGREE OF FINAL POLYNOMIAL MODEL: $\quad 1.000$

Press $<$ ENTER $>$ to continue

Time design matrix for the final model

Time design matrix

$\begin{array}{ll}1.00 & 1.00 \\ 1.00 & 2.00 \\ 1.00 & 3.00 \\ 1.00 & 4.00\end{array}$

Press $<$ ENTER $>$ to continue

Final model coefficients

Press $<$ ENTER $>$ to continue

$\begin{array}{ll}47.35 & 0.5900 \\ 45.85 & 0.6400 \\ 45.45 & 0.7600 \\ 44.15 & 0.7100 \\ 47.20 & 0.5500 \\ 52.25 & 0.3700 \\ 50.45 & 1.120 \\ 48.45 & 0.9000 \\ 46.30 & 2.040 \\ 44.35 & 1.020 \\ 50.95 & 0.2300 \\ 45.35 & 2.480 \\ 51.00 & 0.9600 \\ 47.75 & 0.5200 \\ 48.90 & 0.7400\end{array}$




$\begin{array}{ll}49.90 & 0.8800 \\ 46.30 & 0.7600 \\ 52.95 & 0.6500 \\ 45.75 & 0.7200 \\ 44.20 & 2.020\end{array}$

Press $<$ ENTER $>$ to continue

Estimated average polynomial coefficients:

47.743

0.933

Estimated error variance for an individual observation:

0.193

Press $<$ ENTER $>$ to continue

Estimated dispersion matrix of the polynomial coefficients:

$\begin{array}{rr}7.198 & -0.572 \\ -0.572 & 0.301\end{array}$

Press $<$ ENTER $>$ to continue

Coefficients of average polynomial model and corresponding standard errors and $95 \%$ confidence intervals

\begin{tabular}{lccccc} 
Degree & Coeff & Etd & \multicolumn{2}{c}{$\begin{array}{c}\text { Confidence } \\
\text { Interval }\end{array}$} & \multicolumn{2}{c}{$\begin{array}{c}\text { Half } \\
\text { interval }\end{array}$} \\
0.000 & 47.743 & 0.612 & 46.462 & 49.023 & 1.281 \\
1.000 & 0.933 & 0.130 & 0.660 & 1.206 & 0.273
\end{tabular}

Press $<$ ENTER $>$ to continue

Fitted average curve and corresponding standard errors and $95 \%$ confidence intervals for each time point

\begin{tabular}{lccccc}
$\begin{array}{l}\text { Time } \\
\text { point }\end{array}$ & $\begin{array}{c}\text { Fitted } \\
\text { value }\end{array}$ & $\begin{array}{c}\text { Std } \\
\text { error }\end{array}$ & \multicolumn{2}{c}{$\begin{array}{c}\text { Confidence } \\
\text { interval }\end{array}$} & $\begin{array}{c}\text { Half } \\
\text { interval }\end{array}$ \\
1.000 & 48.676 & 0.828 & 47.243 & 50.108 & 1.433 \\
2.000 & 49.609 & 0.807 & 48.211 & 51.006 & 1.397 \\
3.000 & 50.542 & 0.830 & 49.104 & 51.979 & 1.437 \\
4.000 & 51.475 & 0.894 & 49.927 & 53.022 & 1.547
\end{tabular}

Press $<$ ENTER $>$ to continue

Pressing $<$ ENTER $>$ at this point allows the user to obtain the graphical output described in the text. 